\title{
Wideband High-Gain Millimetre/Submillimetre Wave Antenna Using Additive Manufacturing
}

\author{
Chao $\mathrm{Gu}^{1}$, Steven Gao ${ }^{1 *}$, Benito Sanz Izquierdo ${ }^{1}$, Greg Gibbons ${ }^{2}$, Paul R. Young ${ }^{1}$, Edward A. Parker ${ }^{1}$, \\ Ying Liu ${ }^{3}$, Geyi Wen ${ }^{4}$, Youlin Geng 5 , Zhiqun Cheng ${ }^{5}$ \\ ${ }^{1}$ School of Engineering and Digital Arts, University of Kent, Canterbury, UK \\ ${ }^{2}$ WMG, International Manufacturing Centre, University of Warwick, Coventry, UK \\ ${ }^{3}$ Science and Technology on Antenna and Microwave Laboratory, Xidian University, Xi' an 710071, China \\ ${ }^{4}$ Research Center of Applied Electromagnetics, Nanjing University of Information Science and Technology, \\ Nanjing, China \\ ${ }^{5}$ Key Lab of RF Circuits and Systems of the Ministry of Education of China, Hangzhou Dianzi University, \\ Hangzhou 310018, China \\ *s.gao@kent.ac.uk
}

\begin{abstract}
This paper presents a novel design of a wideband high-gain resonant cavity antenna (RCA) for millimetre and submillimetre wave bands, and its fabrication using additive manufacturing. The proposed RCA antenna consists of a partially reflecting surface and three impedance matching layers fed by a waveguide. Additive manufacturing (AM) techniques are utilized to fabricate the design operating at $30 \mathrm{GHz}$. Two fabrication techniques are assessed for printing the antenna. The first technique is based on printing a dielectric material and fully coating the parts with a metallic layer, while the second technique involves printing the parts in a single process using metal 3D printing. The first technique offers a lightweight solution while the second technique can print the whole model in one run. The antenna design is investigated by both simulations and experiments. The measured results show an $3 \mathrm{~dB}$ gain bandwidth of about $10 \%$, and high gain over $15 \mathrm{dBi}$ for all the three resulting antennas. Good agreement between simulation and measurement is obtained. The antenna has a low cost and achieved good performance in terms of wide bandwidth and high gain, thus it is potentially useful for highspeed wireless communications at millimetre-wave and sub-millimetre-wave frequencies.
\end{abstract}

\section{Introduction}

The increased interest in millimetre and terahertz waves has led to the continuous development of many novel devices operating from millimetre-wave $(\mathrm{mmW})$ to $\mathrm{THz}$ frequencies for a wide range of applications including noninvasive imaging, hazardous material detection, remote sensing, and radio astronomy [1], [2]. For wireless communications, the short wavelengths of $\mathrm{mmW}(1-10 \mathrm{~mm})$ and $\mathrm{THz}(30 \mathrm{~mm}-1 \mu \mathrm{m})$ enable the transmission of vast amounts of data at high data rates [3], [4]. Thus, mmW and $\mathrm{THz}$ bands can be exploited to meet the capacity and latency goals of the next generation wireless links. As the key components of wireless systems, antennas at $\mathrm{mmW}$ and $\mathrm{THz}$ regimes still need further development [5], [6]. There are a number of challenges to be tackled, such as the fabrication of fine features, repeatable dimensional tolerance, surface roughness, and fabrication costs. Traditionally, lenses, horns, and parabolic antennas are used because of their excellent and stable performance [7]. Manufacturing of the aforementioned antennas typically requires computerized numerically controlled (CNC) milling. Alternative antenna fabrication techniques at $\mathrm{mmW}$ and $\mathrm{THz}$ bands have yet to be thoroughly explored.

Previously, microwave wideband antennas have been widely investigated [8]-[14]. These designs all require printed circuit board (PCB) fabrication technique. To push towards higher frequencies, accurately etching the small features of the slots, feedlines, notches, etc. is a major limiting factor for the antenna performance. Resonant cavity antennas (RCAs) can be a solution to the design of $\mathrm{mmW}$ and $\mathrm{THz}$ antenna. Basically, an RCA consists of a radiator with a ground plane in front of a partially reflecting surface (PRS). The broadside gain of the simple radiator can be significantly increased when the reflection amplitude of the PRS is maximized, and the spacing (between the PRS and the ground plane) is optimized to form in-phase wave transmission through the PRS superstrate. Depending on the design of the superstrate layer, the gain bandwidth of RCAs can be improved without much compromise of the maximum achievable gain. Though much work has been done at microwave bands [15]-[18], few RCAs operating at frequencies higher than $30 \mathrm{GHz}$ have yet been proposed [19]-[22]. Authors in [19] describe a V band RCA consisting of a frequency-selective-surface (FSS) over a planar slot-dipole feed. The measured and simulated gain of the antenna is around $11 \mathrm{dBi}$ and $13 \mathrm{dBi}$, respectively. The $3 \mathrm{~dB}$ gain bandwidth is from 61.6 to $64.3 \mathrm{GHz}$. [20] presents a $60 \mathrm{GHz}$ RCA using printed circuit board technology. The compact PRS is fed by an inset-fed microstrip patch antenna. The measured results show a $4.6 \mathrm{GHz} 3 \mathrm{~dB}$ gain bandwidth from 57.6 to $62.2 \mathrm{GHz}$ with a peak gain of $16.4 \mathrm{dBi}$ at $60 \mathrm{GHz}$. A wideband RCA operating at $60 \mathrm{GHz}$ is proposed in [21], where a dual-layer printed FSS-based superstrate is implemented to have a positive gradient. The measurements show the antenna has a $12.2 \% 3 \mathrm{~dB}$ gain bandwidth and a maximum gain of $15.6 \mathrm{~dB}$ at $62.7 \mathrm{GHz}$. It is worth mentioning that the design freedom of the above works is greatly limited by the thickness of the commercially available substrates and spacers. In addition, the dielectric substrate loss at higher frequencies is considerable.

To overcome the drawbacks using conventional PCB techniques, additive manufacturing (AM) can be used to fulfill $\mathrm{mmW}$ and $\mathrm{THz}$ antenna development tasks [23]-[25]. AM is becoming a popular technology for product development and manufacturing operations. Known also as three-dimensional printing (3DP), AM builds arbitrarily 
shaped 3D objects by depositing plastic or metal material layer by layer. Compared with traditional manufacturing processes, AM can offer the mass production of complicated parts with reduced lead-time. For the RF/microwave industry, the use of AM allows the development of bespoke RF components that can achieve reduced size, weight and lead times [26]. To date, a wide range of 3DP technologies have been developed, all with different advantages and disadvantages in terms of resolution, speed, surface quality, material choice, geometric limits, and cost [27]. Generally, material extrusion (e.g. fused deposition modelling - FDM), Powder Bed Fusion (e.g. selective laser sintering - SLS) and material jetting (e.g. multi-jet modelling - MJM) are the dominant techniques for printing dielectric geometries, while Powder Bed Fusion (laser or electron beam) and metal binder jetting are the most common methods to print metallic structures.

This paper reports a novel design of $\mathrm{mmW}$ and low $\mathrm{THz}$ RCAs that can be fabricated using different 3DP technologies, i.e., metal binder jetting and MJM. For the metal binder jetting method, the entire antenna can be printed in metal, so there is no need to manually assemble the antenna parts after printing other than attaching a waveguide to coax adapter. Two further antenna prototypes have been built using two material and MJM process variants for the dielectric printing approach followed by an electroplating process. Measurement and numerical results show good agreement for the three resulting antennas. The remainder of this paper is organised as follows. Section 2 describes the antenna design procedures and full-wave simulation results. Section 3 illustrates the fabrication processes and experimental results. Finally, conclusions are made in Section 4.

\section{Design of the RCA antenna}

For illustration, a typical RCA antenna consists of a PRS layer, a PEC plane, and a microstrip patch antenna as the feed. The antenna operation principle can be explained by the ray-tracing analysis in [15]. The EM wave is emitted from the feed antenna and undergoes multiple reflections between the PRS and the ground. The distance between the PRS and ground plane $h$ required for maximum broadside directivity at the resonance frequency $f_{r}$ can be formulated as

$h=\frac{c}{4 \pi f_{r}}\left(\varphi_{P R S}+\varphi_{G N D}-2 N \pi\right), N=0,1,2, \ldots$

where $\varphi_{P R S}$ and $\varphi_{G N D}$ are the phases of reflection coefficients of the PRS and the ground, respectively. For a PEC ground plane, its reflection phase is a multiple of $\pi$, while the reflection phase of the PRS normally varies with the frequency. According to the resonant condition illustrated by Equation (1), at the given frequency, the cavity height $h$ can be derived as long as the value of $\varphi_{P R S}$ is known.

The first step is to design the PRS layer that is compatible with available AM techniques. The PRS is essentially a periodic structure that can exhibit high reflectivity at the desired frequency, so the metallic grid FSS is an ideal candidate to be produced by AM. Fig. 1(a) shows the PRS unit cell with its geometrical parameters. The material of the hollow square is copper and the periodicity of the unit cell $P=4.8 \mathrm{~mm}$. The inner lateral dimension and thickness are $4 \mathrm{~mm}$ and $1 \mathrm{~mm}$, respectively. $\mathrm{CST}^{\mathrm{TM}}$ Microwave Studio is used to characterize the unit cell. By applying a Floquet-Port setup, the scattering parameters can be calculated under plane wave illumination. The simulated magnitude and phase of the reflection coefficient are shown in Fig. 1(b). To maintain a high directivity, high reflectivity is required inside the antenna cavity. However, the $3 \mathrm{~dB}$ gain bandwidth reduces as the magnitude of $S_{11}$ increases.

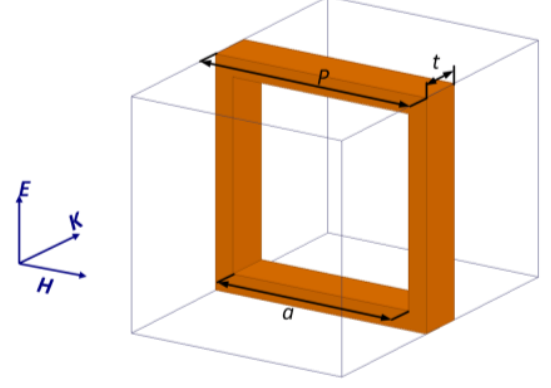

$a$

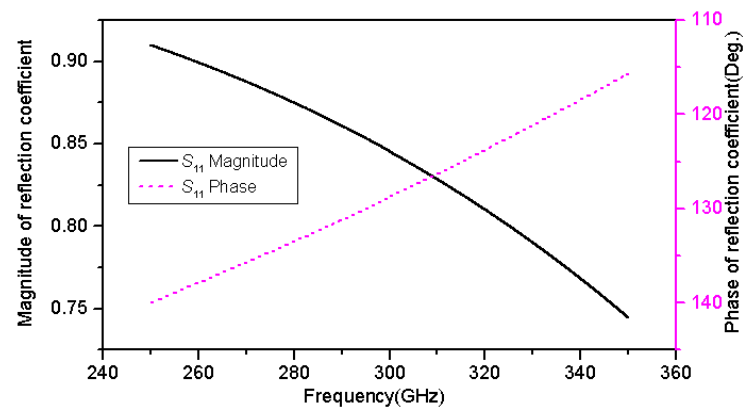

$b$

Fig. 1. Unit cell model of the PRS under normal incidence and the simulated results of the unit cell using CST

(a) Dimensions of the unit cell constituting the PRS layer of the RCA antenna, (b) Magnitude and phase response of the simulated reflection coefficient

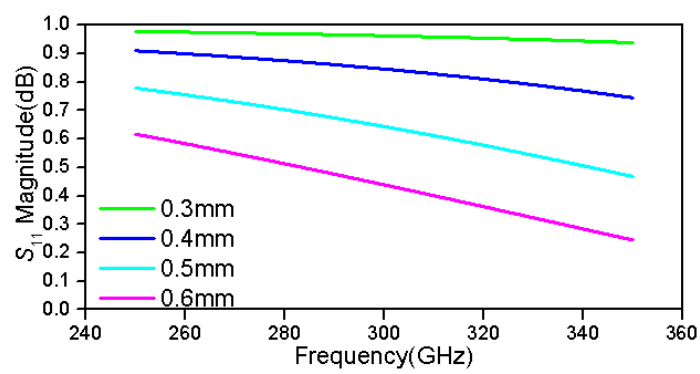

(a)

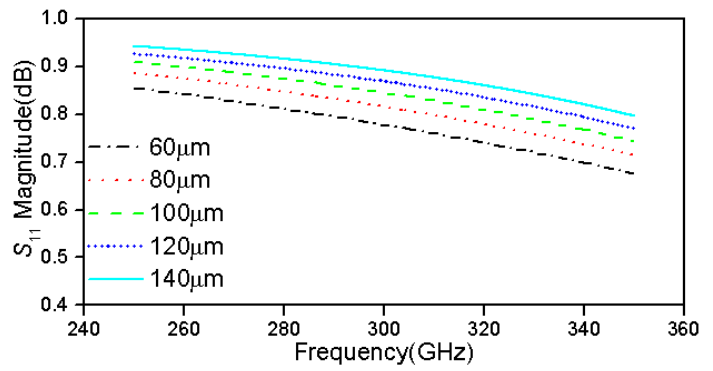

(b)

Fig. 2. Parametric study of unit cell (a) lateral size " $a$ ”; (b) thickness" " $t$ "

So the design is constrained within a trade-off between the peak gain and $3 \mathrm{~dB}$ bandwidth. Parametric studies of the unit cell dimensions are shown in Fig. 2. The two figures indicate that decreasing the size of the hollow area or increasing the thickness of the unit cell can lead to the higher reflectivity, while the former has a larger influence than the latter. Given 
the phase value at the operating frequency, according to (1), the PRS height $h$ can be calculated and optimized to have the best boresight directivity. the transition layers, the antenna impedance mismatches almost in the whole frequency range, while a $-10 \mathrm{~dB} S_{11}$ bandwidth from $288 \mathrm{GHz}$ to $318 \mathrm{GHz}$ is obtained with the

Table 1 Antenna design parameters ( $\mathrm{mm}$ )

\begin{tabular}{|c|c|c|c|c|c|c|c|c|c|c|c|c|c|c|c|}
\hline$h$ & $t_{l}$ & $t_{2}$ & $t_{3}$ & $l_{s}$ & $w_{s}$ & $d_{l}$ & $d_{2}$ & $l$ & $w$ & $w_{a}$ & $w_{b}$ & $w_{c}$ & $w_{d}$ & $w_{e}$ & $w_{f}$ \\
\hline 0.44 & 0.1 & 0.1 & 0.02 & 0.7 & 0.25 & 0.85 & 0.6 & 0.44 & 0.2 & 0.43 & 0.86 & 0.12 & 0.11 & 0.63 & 0.11 \\
\hline
\end{tabular}

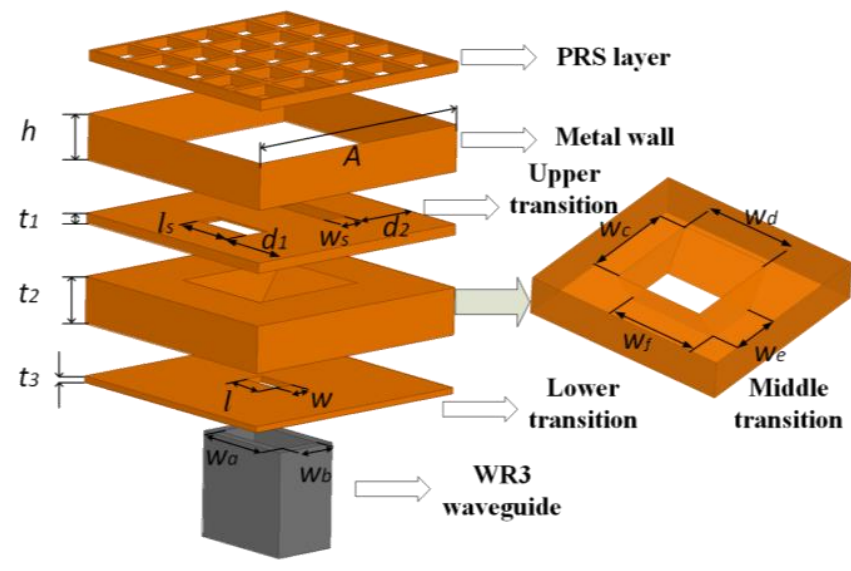

Fig. 3. Detailed antenna configurations

As shown in Fig 3, the complete RCA is composed of a $5 \times 5$ metal PRS superstate, three transitions, and a WR3 waveguide feed. Note that the metal wall is used to support the PRS layer and for ease of fabrication. Normally, direct illumination of the PRS leads to impedance mismatching, lowering the antenna efficiency. Thus, we propose a novel impedance matching technique that consists of three transition layers. The lower transition layer contains a small slot excited by the waveguide port. The middle layer is a thick cavity covered by the upper transition layer where two identical, symmetrical parallel slots are used to broaden the impedance matching bandwidth of the single slot feed. The transition layer generates additional modes at the frequencies of 290 and $310 \mathrm{GHz}$, respectively when the two parallel slots are simultaneously excited.

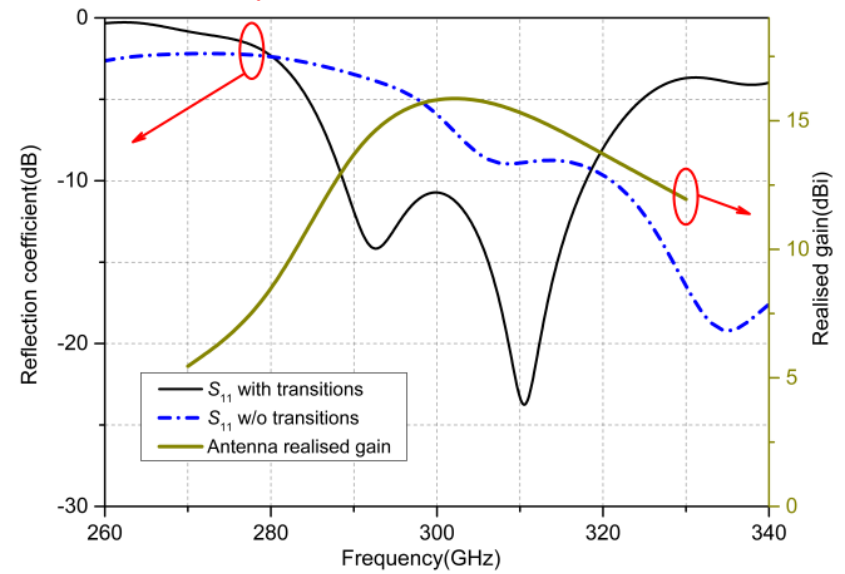

Fig. 4. Simulated reflection coefficient and gain

After careful optimisation of the parameters of the three impedance matching layers, the full-wave simulation results of the antenna performance are obtained. Table 1 shows the antenna geometric parameters. As shown in Fig. 4, without

transitions. The peak realised gain is $16.5 \mathrm{dBi}$ at $300 \mathrm{GHz}$.
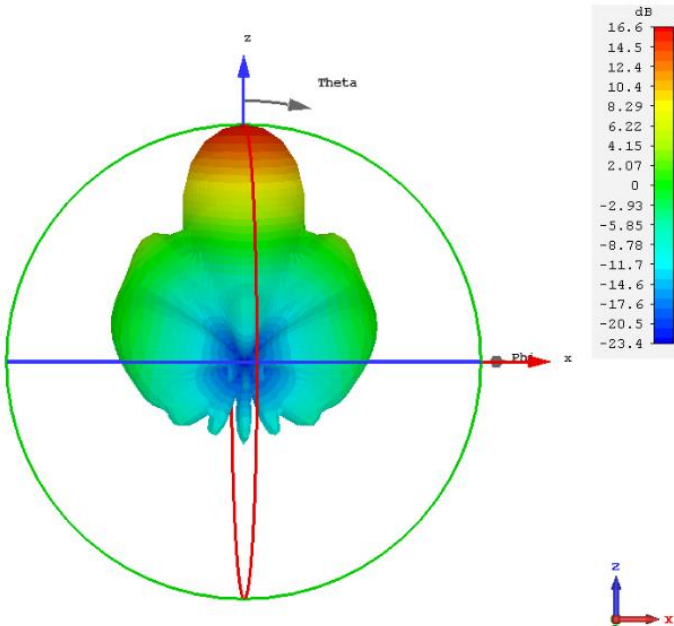

(a)

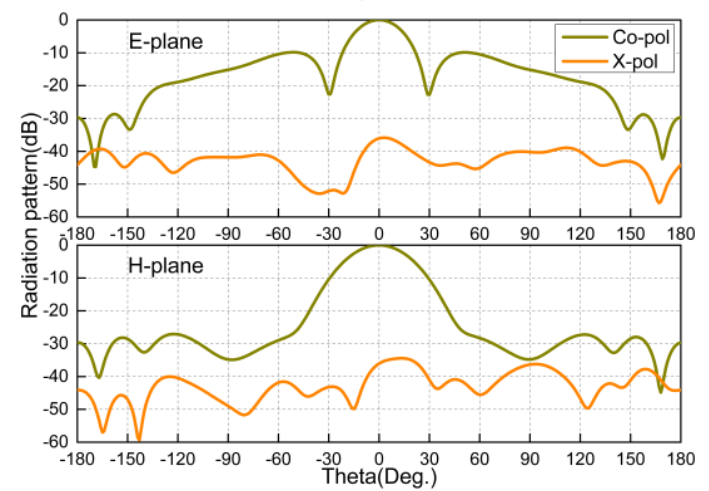

(b)

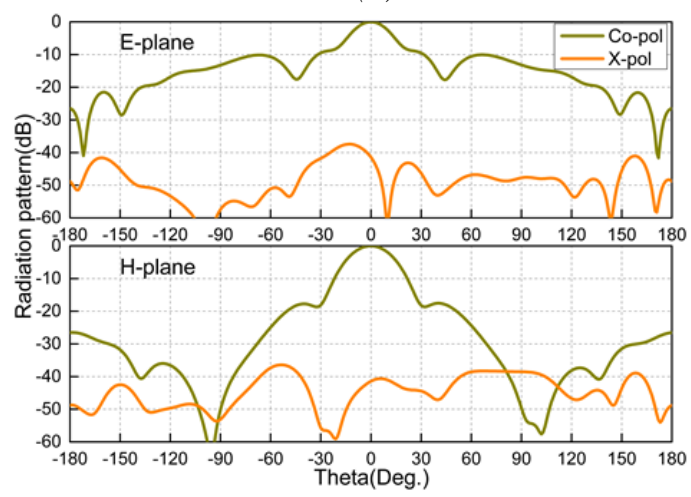

(c) 


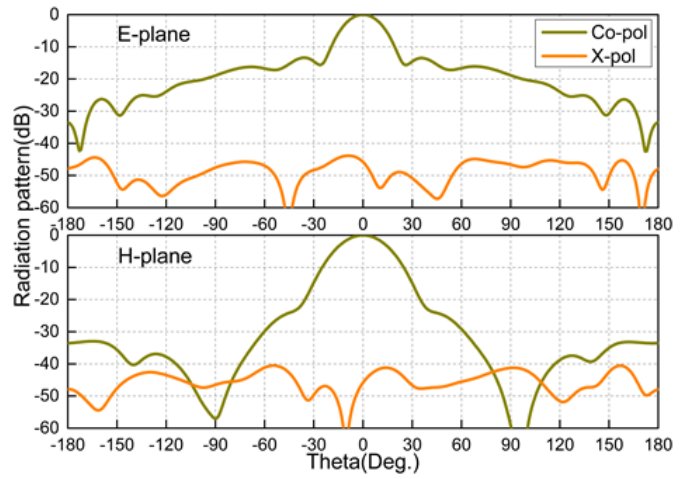

(d)

Fig. 5. Siulated radiation patterns (a) $3 D$ pattern at 300 $\mathrm{GHz}$; (b) $290 \mathrm{GHz}$; (b) $300 \mathrm{GHz}$; (d) $315 \mathrm{GHz}$

Fig. 5 illustrates the simulated $3 \mathrm{D}$ radiation pattern at 300 $\mathrm{GHz}$ and normalized ration patterns at 290, 300, and $315 \mathrm{GHz}$. The plots show directive beams pointing at broadside in the band of interest. Note that we use a metal wall to support the PRS superstate. This will be beneficial to the 3D printing of the antenna structure. Furthermore, the metallic wall improves the antenna gain by $2 \mathrm{dBi}$ and $S_{11}$ is also slightly better using the metal support, as shown in Fig. 6. A possible disadvantage is that the side lobe level of the radiation patterns could be increased due to the generated resonant modes in the metal cavity.

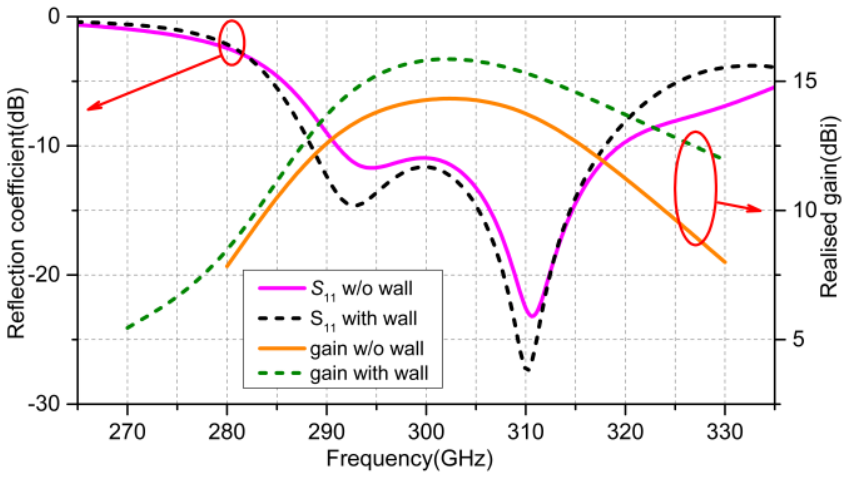

Fig. 6. Effect of the supporting metal wall

Based on the above $300 \mathrm{GHz}$ RCA analysis, a scaled design operating at $30 \mathrm{GHz}$ was simulated. It is straightforward to work out the antenna dimensions required as the whole antenna is made of full metal. Therefore, all the values of the antenna design parameters are 10 times larger than those at $300 \mathrm{GHz}$ given in Table 1 . The only difference is that the feeding waveguide is WR28, with the port dimensions of $7.112 \times 3.556 \mathrm{~mm}$ working at $26.5-40 \mathrm{GHz}$. Fig. 7 shows the scaled $30 \mathrm{GHz}$ version of the antenna configuration in Fig. 3. It can be seen from Fig. 8(a), the better than $-10 \mathrm{~dB} S_{11}$ performance ranges from $29 \mathrm{GHz}$ to $32.3 \mathrm{GHz}$ with a maximum gain of $16.2 \mathrm{dBi}$ in the broadside direction. Fig. 8(b) depicts the simulated $\mathrm{E}$ - and $\mathrm{H}$-plane radiation patterns. Compared to the $300 \mathrm{GHz}$ radiation patterns in Fig. 5, they follow the same trend.

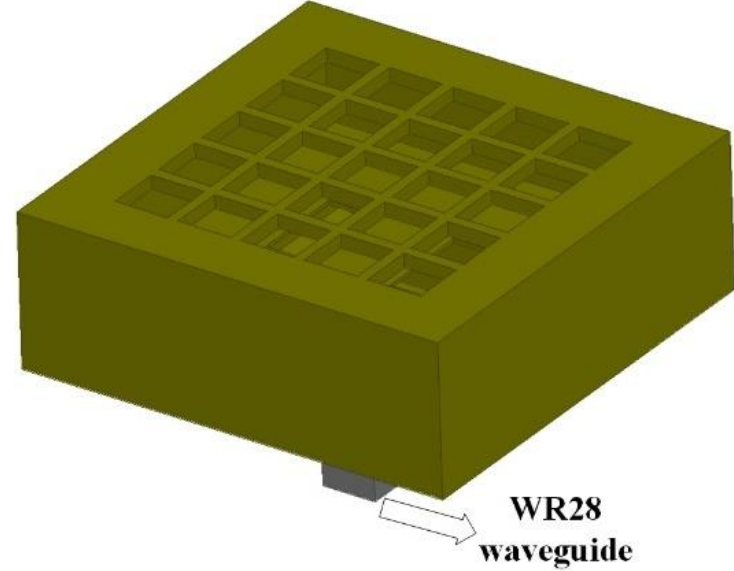

Fig. 7. Perspective view of the $30 \mathrm{GHz}$ design

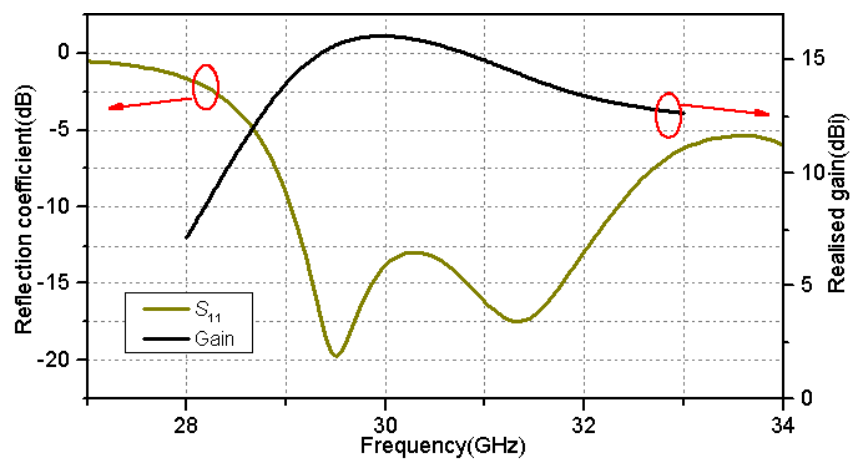

(a)

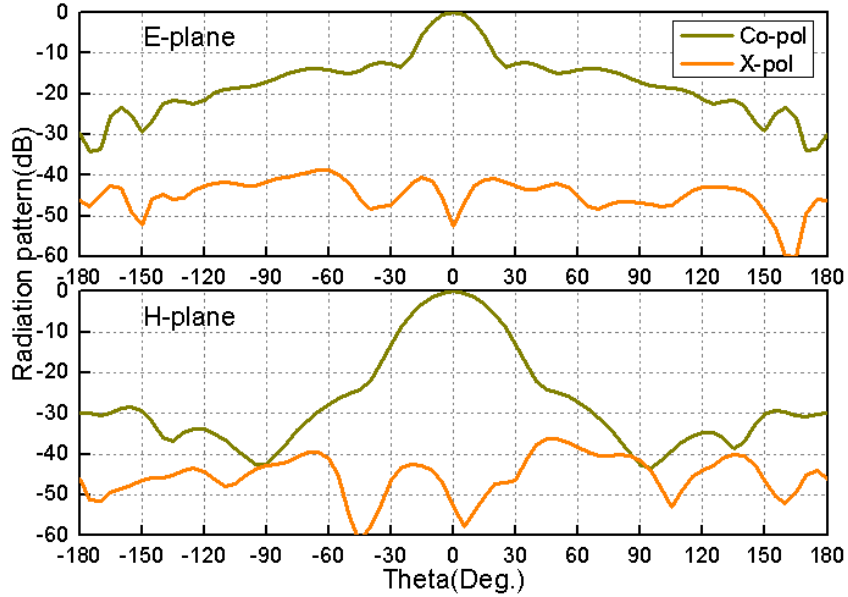

(b)

Fig. 8. Simulated (a) reflection coefficient and gain; (b) Eand $\mathrm{H}$-plane patterns at $30 \mathrm{GHz}$

\section{3D Printing of RCAs}

Fabrication of the above designs requires highresolution printers. Unfortunately, for the $300 \mathrm{GHz} \mathrm{RCA}$ design, almost none of the available AM techniques are capable of printing such fine details of the proposed antennas. It is more reasonable to focus on the $30 \mathrm{GHz}$ design using both dielectric and metal printing. The detailed fabrication processes of the two approaches are introduced in this section.

\section{1. $3 D$ dielectric printing using material jetting}

The polymeric antenna components were printed using two different commercial multi-jet modellers, J750 (Stratasys Inc, USA) and Projet MJP 2500+ (3D Systems Inc, USA). Multijet Modeller (MJM) technology manufactures parts by the ink-jet deposition of UV curable polymers. Each 
deposited layer of resin is instantaneously solidified by exposure to a UV lamp following the ink-jet print head. A counter-rotating roller in front of the print head ensures that each layer is the desired thickness prior to UV cure. The J750 system was selected as it offers high resolution (14 $\mu \mathrm{m}$ in the $\mathrm{z}$-axis, $42 \mu \mathrm{m} \mathrm{x}$ and $\mathrm{y}$-axis), high accuracy (down to $20 \mu \mathrm{m}$ ) and good surface finish. The MJP 2500+ offers a slightly lower resolution $(32 \mu \mathrm{m} \times 28 \mu \mathrm{m} \times 32 \mu \mathrm{m})$ but, due to the rapid solidification of the resins after deposition, provides sharper edge detail than the $\mathbf{J} 750$.

The build materials used were (J750:- VeroPureWhite RGD837 - Stratasys Inc, USA), (MJP 2500+:- Visijet M2 RWT - 3D Systems Inc, USA). These are both diacrylate based polymers containing tricyclodecane dimethanol diacrylate, other acrylate monomers, and $\mathrm{TiO}_{2}$ as a filler. If the fabrication is for the applications above $100 \mathrm{GHz}$, a 3-D Systems Viper si2 with Accura Xtreme resin would be the suitable material as demonstrated in [28].

The parts were built using industry default parameters which represent a $14 \mu \mathrm{m}(\mathrm{J} 750)$ and $32 \mu \mathrm{m} \mathrm{Z}$-axis resolution (MJP 2500+). During the build process, the parts were supported using SUP705 (J750) and Visijet M2 SUP (MJP $2500+$ ) support material (acrylic based). The parts were built with the smallest dimension in the vertical $(\mathrm{z})$ direction.

After manufacture, the samples were manually removed from the build plate, and the support material was removed using (J750) a high-pressure water jet (Genie Pro600 Wash Station, Gemini Cleaning Systems Ltd, UK), (MJP 2500+) a steam bath (MJP EasyClean, 3D Systems Inc, USA). Parts from both systems were then air dried. The fabricated antenna parts are shown in Fig. 9 (a).

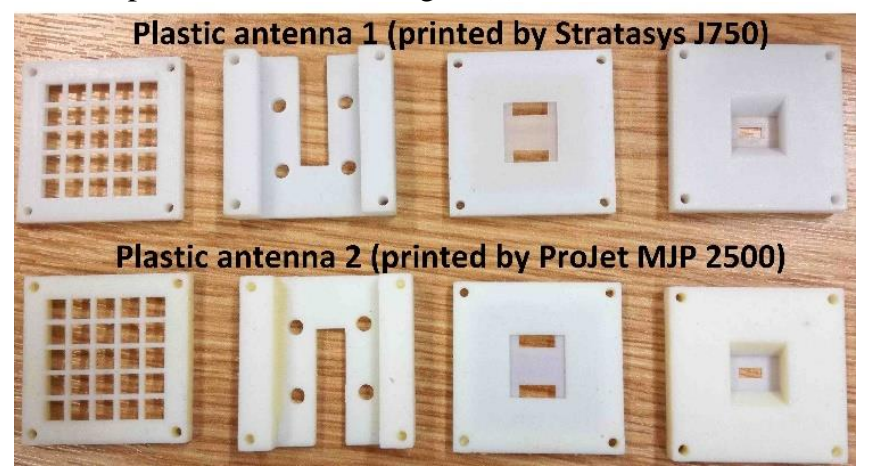

(a)

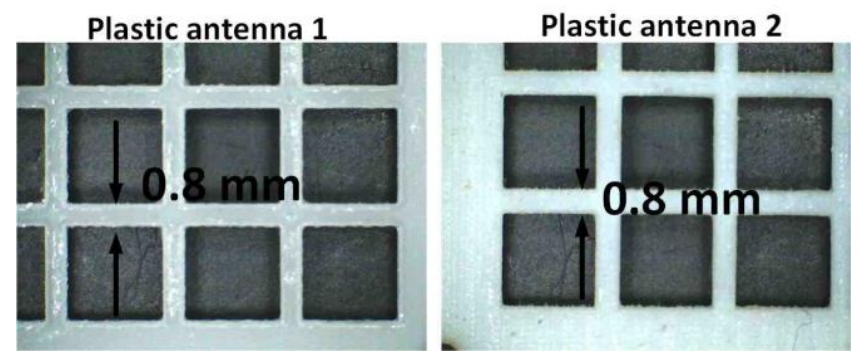

(b)

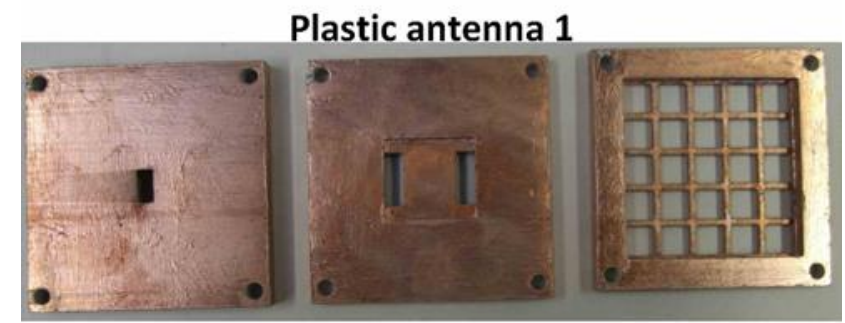

Plastic antenna 2

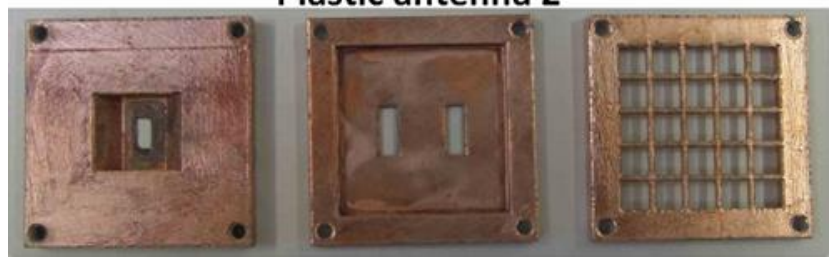

(c)

Fig. 9. Photos of (a) $3 D$ printed polymeric parts; (b) polymeric PRS surface detail; (c) copper plated parts

The next step to create a functional antenna using the printed plastic parts is metallization. The surface roughness of the parts is an important parameter. At $\mathrm{mmW}$ and $\mathrm{THz}$ frequencies, surface roughness tends to become on the order of the skin depth, resulting in attenuation of transmission lines. It is, therefore, very important to deposit the metal layer as smoothly as possible and thick enough to ensure the antenna performance. Here, we adopt a two-step procedure which is easy to implement at laboratory level. First, the surfaces of the printed plastic parts should be cleaned with isopropyl alcohol. A commercial silver conductive paint is then evenly spread over the parts by a fine brush. This step is to ensure the surface has a conductive seed layer essential for the subsequent copper deposition. Then the parts are immersed in a tank of copper sulphate and connected to a DC power supply. A 100-mA current for 5 minutes was found to be suitable for depositing a uniform copper layer (approximately $10 \mu \mathrm{m}$ thick). The plated parts for the two antennas are shown in Fig. 9(c). The thickness of the coated copper layer is larger than 10 times of skin depth at $30 \mathrm{GHz}$. So conductor loss is small, provided that the coated copper layer is even.

\subsection{D metal printing using binder jetting}

With metal AM, there is no need to electroplate the parts. The most active advantage is that the entire antenna structure can be printed in one go, thus saving time and labour The technique we use is metal binder jetting. After the STL (3D representation of the part) file is sent to the $3 \mathrm{D}$ printer, the object is built by spreading layers of stainless steel powder (316L) using high-precision binder jetting. A special print head deposits binding agent at specific points. Each layer of powder is dried and spread from the bottom up till the part is completed. The print is sintered in an oven at $1300^{\circ} \mathrm{C}$ for strength. 316L high-grade stainless steel is cost effective and suitable for the significant levels of detail and strength. One can achieve a minimum wall thickness of $0.3 \mathrm{~mm}$ and a minimum detail of $0.1 \mathrm{~mm}$. The printed metal parts and microscopy photo of the metal surface are shown in Fig. 10. 


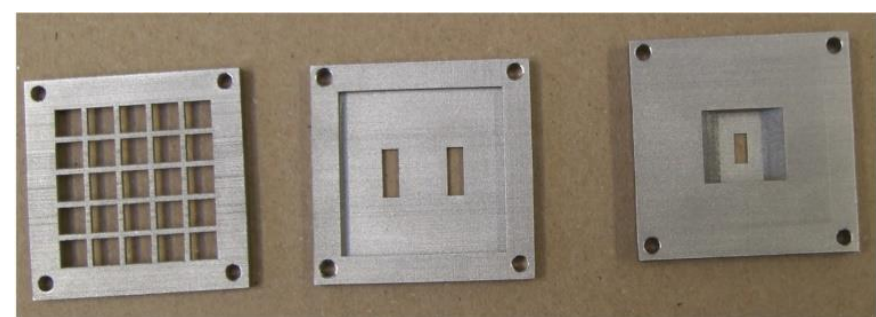

(a)

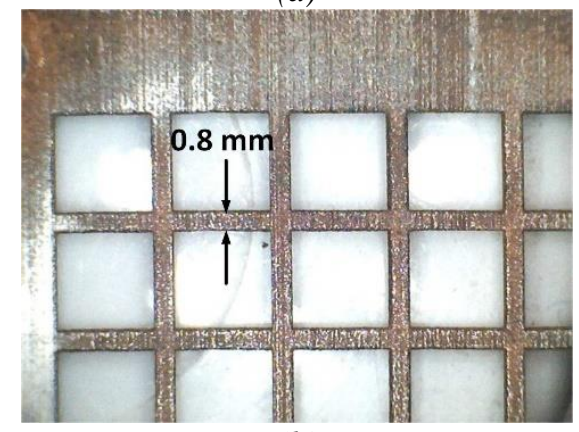

(b)

Fig. 10. Photos of (a) 3D metal printed parts; (b) metal PRS surface

\section{Antenna measurement and results}

Three antenna prototypes have been manufactured using the methods described in Section 3. Measurements were performed in the university's antenna anechoic chamber to verify the antenna design concept. To measure the antennas using the Anritsu 37397C VNA, a K-type coaxial cable together with a Flann Microwave WR28 adapter was used. The fabricated RCAs are shown in Fig.11 (a), (b). All the antennas were printed into three component parts to investigate the capabilities of the employed AM techniques, e.g., surface roughness, fabrication tolerance, etc. To measure the antenna performance, a waveguide to coaxial cable connector is used to attach to the assembled antenna.

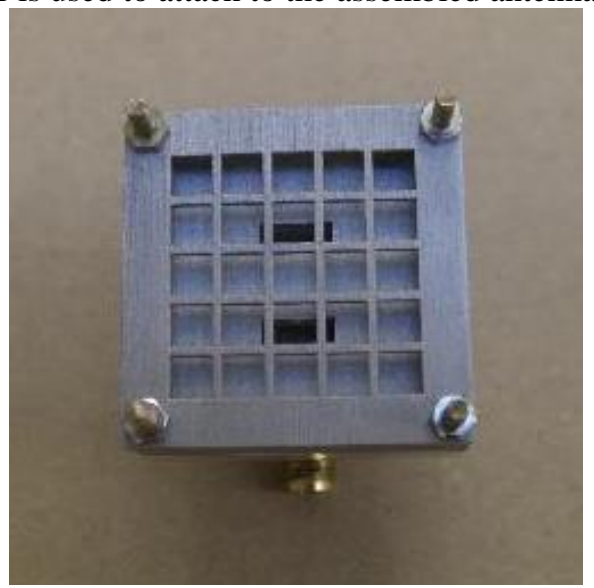

(a)

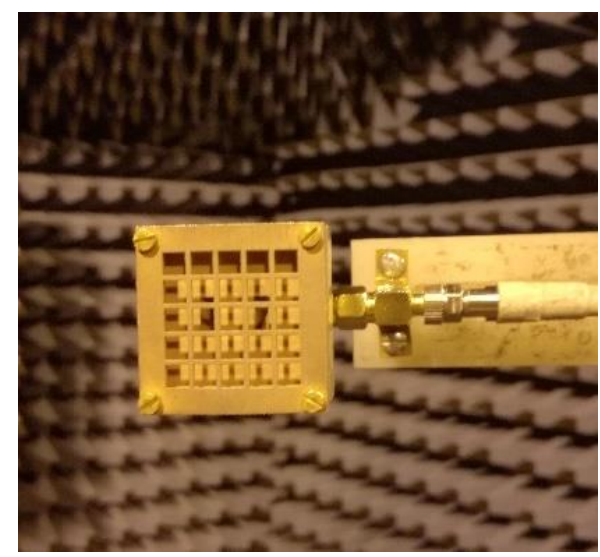

(b)

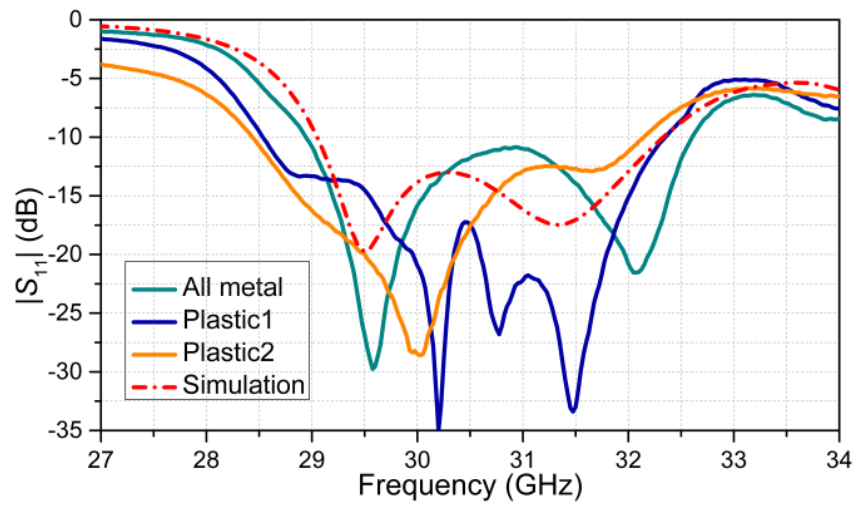

(c)

Fig. 11. Assembled antenna prototypes (a) metal printed; (b) dielectric printed; (c) measured reflection coefficient

The measured reflection coefficients of all three prototypes are shown in Fig. 11(b). It can be observed that the measured $S_{11}$ results are in good agreement with the simulated ones, all with a larger than $3 \mathrm{GHz} S_{11} \leq-10 \mathrm{~dB}$ bandwidth. However, one can notice the differences between the curves which are most likely due to the non-consistent geometry tolerance between different manufacturing processes using different materials. Also, the printing defects can be a reason why $S_{11}$ is sensitive to the printing process. The other factor that can affect $S_{11}$ is the accuracy of the attachment of the waveguide-coax adapter to the antenna radiating parts.

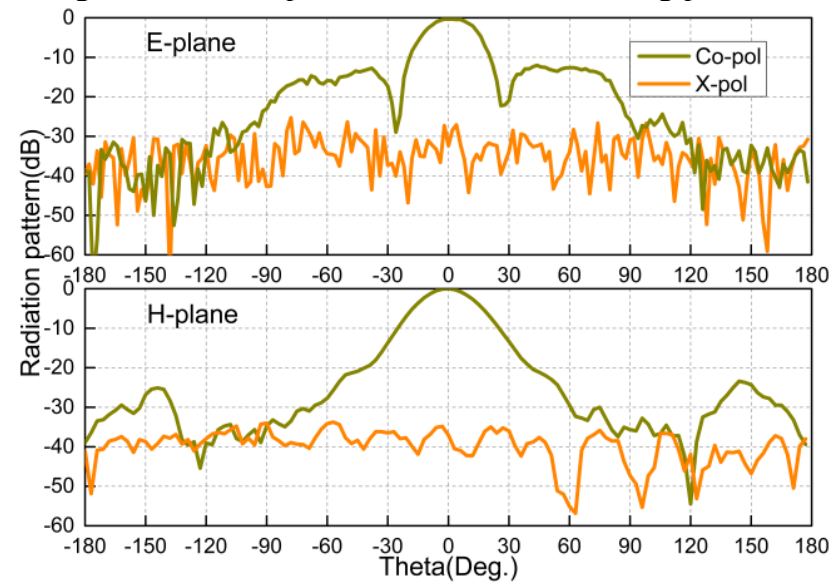

(a) 


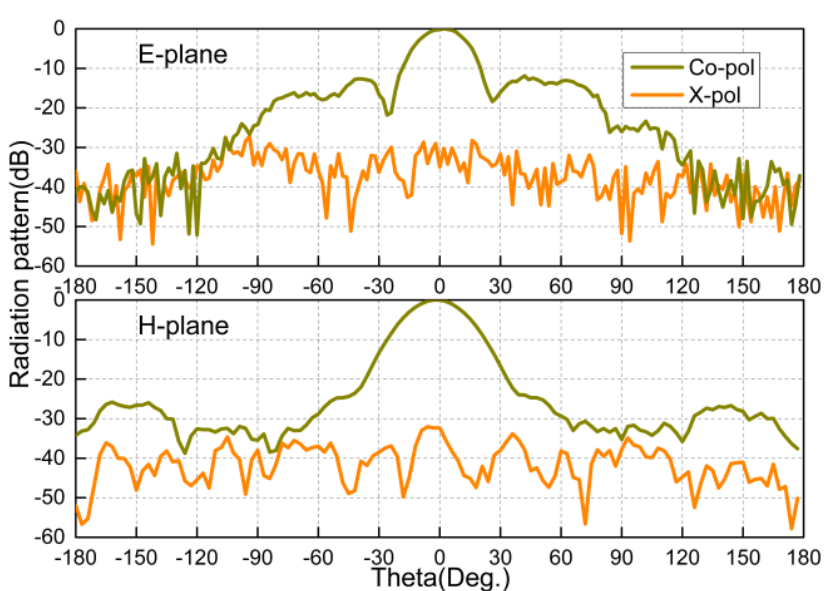

(b)

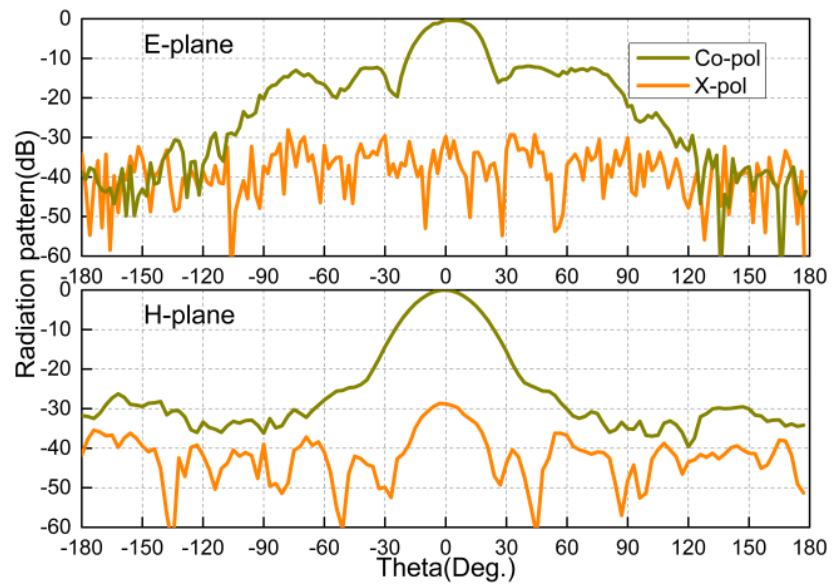

(c)

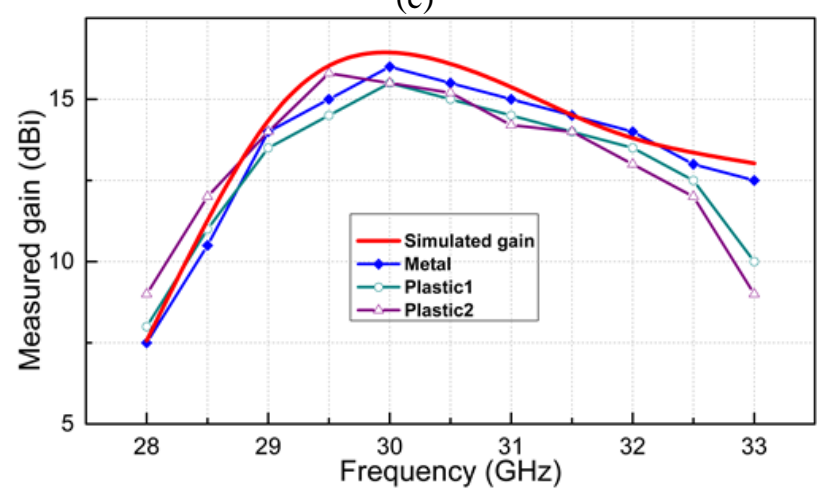

Fig. 12. Measured (a) metal prototype at 30GHz; (b) plasticl at 30GHz; (c) plastic 2 at $30 \mathrm{GHz}$ (d) gain comparison between the measurement and simulation

The antenna radiation performance is validated by the radiation pattern measurement shown in Fig. 12. As expected in Section 2, the measured radiation patterns for the three antennas all show directive main beams pointing in the broadside direction. The cross-polarization levels are better than $30 \mathrm{~dB}$ in the direction of maximum radiation. Measured sidelobe levels (SLL) remain below $-10 \mathrm{~dB}$, although there is a slight deviation between the E-planes of the three plots. Moreover, the $3 \mathrm{~dB}$ beamwidth and the nulls of the simulation and measurement results are in good agreement. Fig. 12(d) shows the measured gain in comparison with the simulated gain. It is clear that all the measured gains of the RCAs are slightly smaller than the simulated one. The gain discrepancy is largely caused by the fabrication tolerance of the three separate parts that were assembled manually. The surface roughness of the copper plated surface is another factor that contributes to the loss. The $3 \mathrm{~dB}$ gain bandwidth of the three RCAs are about $10 \%$ but slightly shifted because of the variation of the antenna impedance matching. It is noted that the key to the wideband RCA is to carefully design the impedance matching as it affects the antenna realized gain. In our design, the most sensitive parameters are $t_{1}$ and $t_{3}$. The parametric study of the two parameters suggests the impedance matching is not deteriorated when the two parameters are varied by $0.2 \mathrm{~mm}$. Furthermore, from the measured results, the proposed antenna has a wide bandwidth so it is not sensitive to small fabrication errors up to $0.2 \mathrm{~mm}$.

Table 2 compares our design and the antennas in the literature. It is clear that the proposed RCA has the smallest effective aperture size using a single PRS layer design. Also, our design is the first millimetre-wave RCA fabricated using viable AM techniques. A comparison for the two used 3DP techniques is provided in Table 3. Both of the two methods are capable of manufacturing parts with a high resolution. The MJM offers the advantages of lightweight plastic parts whereas the MBJ requires an additional sintering process.

Table 2 Comparison of the proposed RCA with the cited works

\begin{tabular}{|c|c|c|c|c|}
\hline Ref & $\begin{array}{c}\text { Fabrication } \\
\text { technique }\end{array}$ & $\begin{array}{c}\text { Lateral } \\
\text { size }\end{array}$ & $\begin{array}{c}3 \mathrm{~dB} \\
\mathrm{BW}\end{array}$ & $\begin{array}{c}\text { Gain } \\
(\mathrm{dBi})\end{array}$ \\
\hline$[9]$ & PCB & $3.1 \lambda_{0}$ & $4 \%$ & 13 \\
\hline$[10]$ & $\mathrm{PCB}$ & $2.8 \lambda_{0}$ & $7.7 \%$ & 16.4 \\
\hline$[11]$ & PCB & $3.6 \lambda_{0}$ & $12.2 \%$ & 16.8 \\
\hline$[12]$ & PCB & $26.7 \lambda_{0}$ & N/A & 15 \\
\hline $\begin{array}{c}\text { This } \\
\text { work }\end{array}$ & $3 \mathrm{DP}$ & $2.4 \lambda_{0}$ & $10 \%$ & 16 \\
\hline
\end{tabular}

Table 3 Comparison of the two 3DP techniques

\begin{tabular}{|c|c|c|c|}
\hline $\begin{array}{c}\text { Fabrication } \\
\text { technique }\end{array}$ & $\begin{array}{c}\text { Manufacturin } \\
\text { g Simplicity }\end{array}$ & $\begin{array}{c}\text { Temperatur } \\
\text { e } \\
\text { Capability }\end{array}$ & Precision \\
\hline $\begin{array}{l}\text { Polymer } \\
\text { Multi-Jet } \\
\text { Modelling } \\
\text { (MJM) }\end{array}$ & $\begin{array}{c}\text { Simple } \\
\text { manufacture } \\
\text { of polymeric } \\
\text { parts } \\
\text { Parts require } \\
\text { metallisation } \\
\text { (surface only) }\end{array}$ & $\begin{array}{c}-\quad \text { Poor } \\
\text { - Polymer } \\
\text { heat } \\
\text { distortion } \\
\text { temperature } \\
\text { typically < } \\
50^{\circ} \mathrm{C} \\
\end{array}$ & $\begin{array}{l}\text {-Excellent } \\
\text { resolution of } \\
50 \mu \mathrm{m} \\
\text {-Excellent } \\
\text { accuracy of } \\
\text { down to } 20 \mu \mathrm{m}\end{array}$ \\
\hline $\begin{array}{c}\text { Metal Binder } \\
\text { Jetting } \\
(\mathrm{MBJ})\end{array}$ & $\begin{array}{c}\text { Simple } \\
\text { manufacture } \\
\text { of metallic } \\
\text { parts } \\
\text {-Two stage } \\
\text { process } \\
\text { requiring } \\
\text { furnace } \\
\text { sintering of } \\
\text { green parts }\end{array}$ & $\begin{array}{l}\text {-Excellent } \\
\text {-Thermal } \\
\text { resistance } \\
\text { of build } \\
\text { metal (e.g } \\
\text { 3161) }\end{array}$ & $\begin{array}{c}\text {-Good } \\
\text { resolution, } \\
\text { typically } 100 \mu \mathrm{m} \\
\text {-Good accuracy } \\
\text { down to } 50 \mu \mathrm{m} \\
\text { for small parts } \\
\text { (higher for } \\
\text { larger parts) }\end{array}$ \\
\hline
\end{tabular}

\section{Conclusion}

Millimeter wave and $\mathrm{THz}$ frequency bands bring challenges to the antenna designer as the frequency increases, the dielectric loss is considerable and designed are limited by the substrate and material (e.g., spacer) choice. Thus, it is 
expected that new designs and fabrication techniques will be developed in these frequency ranges. Inspired by the emerging additive manufacturing processes, a resonant cavity antenna design is proposed operating at $300 \mathrm{GHz}$. To validate the design principle, the antenna has been scaled to $30 \mathrm{GHz}$ and simulated and fabricated by two viable AM techniques, i.e., MJM printing and metal binder jetting. To the best of the authors' knowledge, this is the first paper that compares the fabrication process and antenna performance using the two approaches. The printed dielectric parts are light in weight but need the further process of copper plating, while the metal printed parts can be printed in one run, which is an attractive feature that can reduce the product lead time and cost. In this work, we only utilize metal binder jetting, but there is another viable metal printing technique i.e. Laser Powder Bed Fusion. It has more material options including the low weight Aluminum (AlSi10Mg). This material can be of interest to space applications, where efforts are made to reduce the RF/ antenna system weight. Experimental results show good agreement with the simulated ones, which demonstrates the feasibility of 3D printing the proposed robust antenna design. The slightly shifted impedance matching and gain results are mainly caused by fabrication inaccuracies and waveguide feed misalignment. Overall AM provides more freedom to the design of $\mathrm{mmW}$ and $\mathrm{THz}$ antennas.

\section{Acknowledgments}

The authors would like to thank Mr Richard Hoy from the Laserlines, UK, for support on antenna fabrication. This project is funded by CHIST ERA WISDOM project under EPSRC grant EP/P015840/1, and the EPSRC HVM Catapult Fellowship scheme grant REF: EP/L017121/1.

\section{References}

[1] Rebeiz, G.M.: 'Millimeter-wave and terahertz integrated circuit antennas', Proceedings of the IEEE, 1992, 80, (11), pp. 1748-1770.

[2] Siegel, P.H.: 'Terahertz technology', IEEE Transactions on Microwave Theory and Techniques, 2002, 50, (3), pp. 910-928.

[3] Piesiewicz, R., Kleine-Ostmann, T., Krumbholz, N., et al.: 'Short-Range Ultra-Broadband Terahertz Communications: Concepts and Perspectives', IEEE Antennas and Propagation Magazine, 2007, 49, (6), pp. 2439.

[4] Rappaport, T.S., Shu Sun, Mayzus, R., et al.: 'Millimeter Wave Mobile Communications for 5G Cellular: It Will Work!', IEEE Access, 2013, 1, pp. 335-349.

[5] Gu, C., Gao, S., Sanz-Izquierdo, B.: 'Wideband low-THz antennas for high-speed wireless communications', 2017 IEEE-APS Topical Conf. on Antennas and Propagation in Wireless Communications (APWC), Verona, 2017, pp. 141145 .

[6] Gu, C., Gao, S., Sanz-Izquierdo, B.: 'Low-cost wideband low- $\mathrm{THz}$ antennas for wireless communications and sensing', 2017 10th UK-Europe-China Workshop on Millimetre Waves and Terahertz Technologies (UCMMT), Liverpool, 2017, pp. 1-4.

[7] Imbriale, W.A., Gao, S., Boccia, L.: 'Space antenna handbook' (Wiley \& Sons, 2012).
[8] Kin-Fai Tong, Kwai-Man Luk, Kai-Fong Lee, Lee, R.Q.: 'A broad-band U-slot rectangular patch antenna on a microwave substrate', IEEE Transactions on Antennas and Propagation, 2000, 48, (6), pp. 954-960.

[9] Shi-Chang Gao, Le-Wei Li, Mook-Seng Leong, Tat-Soon Yeo: 'Wide-band microstrip antenna with an H-shaped coupling aperture', IEEE Transactions on Vehicular Technology, 2002, 51, (1), pp. 17-27.

[10] Zhu, F., Gao, S., Ho, A.T., et al.: 'Multiple BandNotched UWB Antenna With Band-Rejected Elements Integrated in the Feed Line', IEEE Transactions on Antennas and Propagation, 2013, 61, (8), pp. 3952-3960.1

[11] Qin, P.-Y., Guo, Y.J., Weily, A.R.: 'Broadband Reflectarray Antenna Using Subwavelength Elements Based on Double Square Meander-Line Rings', IEEE Transactions on Antennas and Propagation, 2016, 64, (1), pp. 378-383.

[12] Novak, M.H., Miranda, F.A., Volakis, J.L.: 'Ultrawideband phased array for small satellite communications', IET Microwaves, Antennas \& Propagation, 2017, 11, (9), pp. 1234-1240.

[13] Liu, H., Liu, Y., Zhang, W., Gao, S.: 'An UltraWideband Horizontally Polarized Omnidirectional Circular Connected Vivaldi Antenna Array', IEEE Transactions on Antennas and Propagation, 2017, 65, (8), pp. 4351-4356.

[14] Sallam, M.O., Kandil, S.M., Volski, V., Vandenbosch, G.A.E., Soliman, E.A.: 'Wideband CPW-Fed Flexible BowTie Slot Antenna for WLAN/WiMax Systems', IEEE Transactions on Antennas and Propagation, 2017, 65, (8), pp. 4274-4277.

[15] Trentini, G.V.: 'Partially reflecting sheet arrays', IRE Transactions on Antennas and Propagation, 1956, 4, (4), pp. 666-671.

[16] Feresidis, A.P., Vardaxoglou, J.C.: 'High gain planar antenna using optimised partially reflective surfaces', IEE Proceedings - Microwaves, Antennas and Propagation, 2001, 148, (6), p. 345.

[17] Wang, N., Liu, Q., Wu C., et al.: 'Wideband Fabry-Perot Resonator Antenna With Two Complementary FSS Layers', IEEE Transactions on Antennas and Propagation, 2014, 62, (5), pp. 2463-2471.

[18] Qin, F., Gao, S., Luo, Q., et al.: 'A Simple Low-Cost Shared-Aperture Dual-Band Dual-Polarized High-Gain Antenna for Synthetic Aperture Radars', IEEE Transactions on Antennas and Propagation, 2016, 64, (7), pp. 2914-2922. [19] Hosseini, A., Capolino, F., De Flaviis, F.: 'Gain Enhancement of a V-Band Antenna Using a Fabry-Pérot Cavity With a Self-Sustained All-Metal Cap With FSS', IEEE Transactions on Antennas and Propagation, 2015, 63, (3), pp. 909-921.

[20] Abbou, D., Vuong, T.P., Touhami, R., Ferrero, F., Hamzaoui, D., Yagoub, M.C.E.: 'High-Gain Wideband Partially Reflecting Surface Antenna for $60 \mathrm{GHz}$ Systems', IEEE Antennas and Wireless Propagation Letters, 2017, 16, pp. 2704-2707.

[21] Attia, H., Abdelghani, M.L., Denidni, T.A.: 'Wideband and High-Gain Millimeter-Wave Antenna Based on FSS Fabry-Perot Cavity', IEEE Transactions on Antennas and Propagation, 2017, 65, (10), pp. 5589-5594.

[22] Gueye, M.B., Ouslimani, H.., Letestu, Y., FanHong, M.: 'Experimental study of $80 \mathrm{GHz}$ Fabry-Pérot cavity antenna based on dual-layer partially reflected surface', Electronics Letters, 2015, 51, (22), pp. 1730-1732. 
[23] Xin, H., Liang, M.: '3-D-Printed Microwave and THz Devices Using Polymer Jetting Techniques', Proceedings of the IEEE, 2017, 105, (4), pp. 737-755.

[24] Cai, F., Chang, Y.-H., Wang, K., Zhang, C., Wang, B., Papapolymerou, J.: 'Low-Loss 3-D Multilayer Transmission Lines and Interconnects Fabricated by Additive Manufacturing Technologies', IEEE Transactions on Microwave Theory and Techniques, 2016, 64, (10), pp. 32083216.

[25] Verploegh, S., Coffey, M., Grossman, E., Popovic, Z.: 'Properties of 50-110-GHz Waveguide Components Fabricated by Metal Additive Manufacturing', IEEE Transactions on Microwave Theory and Techniques, 2017, 65, (12), pp. 5144-5153.

[26] Jun, S.Y., Sanz-Izquierdo, B., Parker, E.A., Bird, D., McClelland, A.: 'Manufacturing Considerations in the 3-D Printing of Fractal Antennas', IEEE Transactions on Components, Packaging and Manufacturing Technology, 2017, 7, (11), pp. 1891-1898.

[27] Calignano, F., Manfredi, D., Ambrosio, E.P., et al.: 'Overview on Additive Manufacturing Technologies', Proceedings of the IEEE, 2017, 105, (4), pp. 593-612.

[28] M. D'Auria et al., "3-D Printed Metal-Pipe Rectangular Waveguides," IEEE Transactions on Components, Packaging and Manufacturing Technology, vol. 5, no. 9, pp. 1339-1349, Sep. 2015. 